\title{
Precoding of Space-Time Block Coded Signals for Joint Transmit-Receive Correlated MIMO Channels
}

\author{
Are Hjørungnes, David Gesbert, and Jabran Akhtar
}

\begin{abstract}
A memoryless linear precoder is designed for orthogonal space-time block codes (OSTBC) for improved performance over block-fading flat correlated Rayleigh fading multiple-input multiple-output (MIMO) channels. Original features of the proposed technique include 1) the precoder can handle both transmit and receive correlation, and 2) the precoder handles any arbitrary joint correlation structure, including the famous so-called non-Kronecker (non-separable) correlation models. The precoder is designed to minimize a symbol error-based metric as function of the joint slowly-varying channel correlation coefficients, which are supposed to be known to the transmitter. Several useful properties of the optimal precoder are given, evidencing the impact of receive correlation on transmitter optimization in certain situations. An iterative fast-converging numerical optimization algorithm is proposed. Monte Carlo simulations over fading channels are used to validate our claims.
\end{abstract}

Index Terms: MIMO, orthogonal space-time block code, precoder optimization, minimum pairwise error probability based metric, power constraint.

\section{INTRODUCTION}

In the area of efficient communications over non-reciprocal MIMO channels, recent research [2], [3], [4] has demonstrated the value of feeding back to the transmitter information about channel state observed at the receiver, especially in non-ideal (that is non-i.i.d.) channel cases. Clearly, the type of feedback may vary largely, depending on its nature, e.g., required rate, instantaneous, or statistical channel state information (CSI), leading to various transmitter design schemes, e.g., [2], [3], [5]. Among those, there has been a growing interest in transmitter schemes that can exploit low-rate long-term statistical CSI

Corresponding author: A. Hjørungnes is with UniK - University Graduate Center, University of Oslo, P. O. Box 70, N-2027 Kjeller, Norway, email: arehj@unik.no, phone +47-64844733, and fax +47-63818146.

J. Akhtar is with the Department of Informatics, University of Oslo, P. O. Box 1080, Blindern, N-0316 Oslo, Norway, email: jabrana@ ifi.uio.no.

D. Gesbert is with the Mobile Communications Department, Eurécom Institute, 2229 Route des Crêtes, BP 193, F-06904 Sophia Antipolis Cédex, France, email: gesbert@eurecom.fr

This work is supported by the Research Council of Norway through project number 157716/432 and by a research contract with Telenor Research AS, Norway. Part of this work was presented in [1]. 
in the form of antenna correlation coefficients. So far, emphasis has been on designing precoders for space-time block coded (STBC) [2] signals or spatially multiplexed streams that are adjusted based on the knowledge of the transmit correlation only while the receiving antennas are uncorrelated [4], [6]. These techniques are well suited to downlink situation where one elevated access point (situated above the surrounding clutter) transmits to a subscriber placed in a rich scattering environment. However, to the best of our knowledge, little attention has been put on the precoding problem in the case where the receiving antennas are correlated. This fact is surprising since the correlated receiver case only depicts a common situation met in the uplink of the corresponding scenario above. More generally, the case where both transmit and receive antennas exhibit some correlation has not been addressed, except implicitly in [3], where it is generally the instantaneous channel state information that is fed back of the transmitter. This can be explained from the widespread belief that detrimental effects caused by receiver correlation cannot be dealt with by the transmitter. However, this is not generally true as we show in this letter.

Here, we assume that only the joint transmit-receive correlation matrix is fed back to the transmitter and we address the question of how to deal with the correlation using a linear precoder posterior to the OSTBC module such that a metric based on the pairwise error probability (PEP) is minimized. We show that, unlike common intuition, the precoder at the transmitter can have a compensating effect on the degradation due to correlation observed at the receiver at least in two important cases: a) There is non-zero receiver correlation and transmitter correlation in the Kronecker model, and b) there is no transmitter correlation but there is receiver correlation, and the overall correlation does not follow the so-called Kronecker model.

Although most previous models for the joint transmit-receive correlation are based on the Kronecker structure [2], the accuracy of these models has recently been questioned in the literature based on measurement campaigns [7]. Therefore, there is interest in investigating the precoding of OSTBC signals for MIMO channels that do not necessarily follow the Kronecker structure. Precoding of uncorrelated channel with mean feedback for quasi-orthogonal codes was considered in [6].

The main contributions of this letter are: (i) We propose a technique in which we minimize certain bounds on the PEP of the OSTBC signal, where the choice of the OSTBC is given in advance. In that respect, our approach is reminiscent of [3], [4], however, our expressions are also valid for the case of a singular correlation matrix and a full correlation matrix. We propose a new, fast converging iterative algorithm which uses the knowledge of the full transmit-receive correlation, regardless of whether the 
Kronecker structure is valid or not. (ii) Some properties of the optimal precoder are found. In the case uncorrelated transmit antennas and an arbitrary number of correlated receive antennas, we show that the optimal precoder boils down to a power allocation strategy across the transmit antennas. We show that in the case that correlation happens to be Kronecker based and the transmit antennas are uncorrelated, then the receive antenna correlation does not have any impact on the precoder design for OSTBC signals, which makes good intuitive sense since in this case every transmit antenna leads to an independent and identically distributed channel vector at the receive array and thus has no reason to be treated differently. In contrast, we point out that if the Kronecker model holds and the transmit antennas are correlated, then the receive correlation does generally play a role in the precoder design and can be compensated for to some extent, both in the Kronecker and non-Kronecker correlation cases.

\section{SYSTEM DESCRIPTION}

\section{A. OSTBC Signal Model}

Figure 1 shows the block MIMO system model. The original bits $b_{i}$ are first modulated into a vector $\boldsymbol{x}=$ $\left[x_{0}, x_{1}, \ldots, x_{K-1}\right]^{T}$ of size $K \times 1$, and where $x_{i} \in \mathcal{A}$, where $\mathcal{A}$ is a signal constellation set such as uniform $M$-PAM, $M$-QAM, or $M$-PSK. $K \log _{2} M$ bits are used to produce the vector $\boldsymbol{x}$. The vector $\boldsymbol{x}$ is transmitted by means of an OSTBC matrix $\boldsymbol{C}(\boldsymbol{x})$ of size $B \times N$, where $B$ and $N$ are the space and time dimension of the given OSTBC, respectively, followed by the precoder $\boldsymbol{F}$ of size $M_{t} \times B$. Then the signal is sent over the MIMO channel $\boldsymbol{H}$ of size $M_{r} \times M_{t}$. The channel is corrupted by the additive block noise $\boldsymbol{V}$, of size $M_{r} \times N$, which is complex Gaussian circularly distributed with independent components having variance $N_{0}$. The $M_{r} \times N$ receive block signal $\boldsymbol{Y}$ becomes

$$
\boldsymbol{Y}=\boldsymbol{H} \boldsymbol{F C}(\boldsymbol{x})+\boldsymbol{V}
$$

The receiver is assumed to know the channel matrix $\boldsymbol{H}$ exactly and it performs a maximum likelihood decoding (MLD) of blocks $\boldsymbol{Y}$ of size $M_{r} \times N$ to find an estimate of $\boldsymbol{x}$ denoted $\hat{\boldsymbol{x}}$. This vector is demodulated into the decoded bits $\hat{b}_{i}$.

Let $E\left[\left|x_{i}\right|^{2}\right]=\sigma_{x}^{2}$. Since a OSTBC is assumed, the following holds

$$
\boldsymbol{C}(\boldsymbol{x}) \boldsymbol{C}^{H}(\boldsymbol{x})=a \sum_{i=0}^{K-1}\left|x_{i}\right|^{2} \boldsymbol{I}_{B},
$$

where $a=1$ if $\boldsymbol{C}(\boldsymbol{x})=\mathcal{G}_{2}^{T}, \boldsymbol{C}(\boldsymbol{x})=\mathcal{H}_{3}^{T}$, or $\boldsymbol{C}(\boldsymbol{x})=\mathcal{H}_{4}^{T}$ in [8] and $a=2$ if $\boldsymbol{C}(\boldsymbol{x})=\mathcal{G}_{3}^{T}$ or $\boldsymbol{C}(\boldsymbol{x})=\mathcal{G}_{4}^{T}$ in [8]. The rate of the code is $K / N$. Any OSTBC can be used. 


\section{B. Arbitrarily Correlated Channel Models}

A block-fading flat correlated Rayleigh fading channel model [2] is assumed. Most previous works consider transmit correlation only, and look at the so-called Kronecker assumption in case of joint transmitreceive correlation. Here, let the channel $\boldsymbol{H}$ have zero mean, complex Gaussian circularly distributed with positive semi-definite autocorrelation given by $\boldsymbol{R}=E\left[\operatorname{vec}(\boldsymbol{H}) \operatorname{vec}^{H}(\boldsymbol{H})\right]$ of size $M_{t} M_{r} \times M_{t} M_{r}$, where the operator $\operatorname{vec}(\cdot)$ stacks the columns of the matrix it is applied to into a long column vector [9]. Let us now examine two possible models for the correlation $\boldsymbol{R}$.

1) Kronecker Model: In the Kronecker model [2], the full autocorrelation matrix $\boldsymbol{R}$ is given by

$$
\boldsymbol{R}=\boldsymbol{R}_{t}^{T} \otimes \boldsymbol{R}_{r}
$$

where the operator $(\cdot)^{T}$ denotes transposition, $\otimes$ is the Kronecker product, the matrices $\boldsymbol{R}_{r}$ and $\boldsymbol{R}_{t}$ are the correlation matrices of the receiver and transmitter, respectively, and their sizes are $M_{r} \times M_{r}$ and $M_{t} \times M_{t}$. This widely used channel model was used in [4], to find a linear precoder when $\boldsymbol{R}_{r}=\boldsymbol{I}_{M_{r}}$.

2) Non-Kronecker Model: The Kronecker model shown in (3) can be interpreted through the idea that the receive (or transmit) correlation does not depend on at which transmit (or receive) antenna the channel measurements are performed. Recently, it was pointed out that this model does not properly describe general propagation scenarios, in which the multipath structure does change depending which transmit antenna is used [7]. Hence, in general, $\boldsymbol{R} \neq \boldsymbol{R}_{t}^{T} \otimes \boldsymbol{R}_{r}$.

Uncorrelated transmit antennas: In the case where the transmit antennas are uncorrelated, the total correlation matrix can be expressed as

$$
\boldsymbol{R}=\left[\begin{array}{cccc}
\boldsymbol{R}_{r_{0}} & \mathbf{0}_{M_{r} \times M_{r}} & \cdots & \mathbf{0}_{M_{r} \times M_{r}} \\
\boldsymbol{0}_{M_{r} \times M_{r}} & \boldsymbol{R}_{r_{1}} & \cdots & \mathbf{0}_{M_{r} \times M_{r}} \\
\vdots & \vdots & \ddots & \vdots \\
\mathbf{0}_{M_{r} \times M_{r}} & \mathbf{0}_{M_{r} \times M_{r}} & \cdots & \boldsymbol{R}_{r_{M_{t}-1}}
\end{array}\right],
$$

where $\boldsymbol{R}_{r_{i}}$ is the receive correlation matrix seen when the signal is launched from transmitter number $i$ and the matrix $\mathbf{0}_{k \times l}$ has size $k \times l$ containing only zeros.

\section{PRoblem Formulation AND PROPERTIES}

\section{A. Optimal Precoder Problem Formulation}

The goal is to find the matrix $\boldsymbol{F}$ such that a PEP based metric is minimized under an appropriate power constraint. The autocorrelation matrix $\boldsymbol{R}$, unlike the instantaneous channel matrix $\boldsymbol{H}$, is assumed to be known, via slow feedback, to the transmitter. 
Using (2), the power constraint on the transmitted block $\boldsymbol{Z}=\boldsymbol{F} \boldsymbol{C}(\boldsymbol{x})$ is given by

$$
a K \sigma_{x}^{2} \operatorname{Tr}\left\{\boldsymbol{F} \boldsymbol{F}^{H}\right\}=P,
$$

where $P$ is the average power used by the transmitted block $Z$.

Let $\boldsymbol{x}_{k}$ and $\boldsymbol{x}_{l}$ be two different realizations of the $K \times 1$ vector $\boldsymbol{x}$. Suppose codeword $\boldsymbol{C}\left(\boldsymbol{x}_{k}\right)$ is transmitted while $\boldsymbol{C}\left(\boldsymbol{x}_{l}\right)$ is detected. Let $\boldsymbol{E}_{k, l}(\boldsymbol{x})=\boldsymbol{C}\left(\boldsymbol{x}_{k}\right)-\boldsymbol{C}\left(\boldsymbol{x}_{l}\right)$ be the error matrix of size $B \times N$. If it is assumed that no mean value nor side information is present, the PEP criterion proposed in [3] can be written as: $\operatorname{det}\left(\boldsymbol{R}^{-1}+\frac{\boldsymbol{F E}_{k, l}(\boldsymbol{x}) \boldsymbol{E}_{k, l}^{H}(\boldsymbol{x}) \boldsymbol{F}^{H}}{4 N_{0}} \otimes \boldsymbol{I}_{M_{r}}\right)$. Unfortunately, this criterion is not able to handle the case of singular $\boldsymbol{R}$. In [4], only transmitter correlation in the Kronecker model was considered and the criterion used was $\operatorname{det}\left(\boldsymbol{I}_{M_{t}}+\frac{1}{4 N_{0}} \boldsymbol{R}_{t}^{1 / 2} \boldsymbol{F} \boldsymbol{E} \boldsymbol{E}^{H} \boldsymbol{F}^{H} \boldsymbol{R}_{t}^{1 / 2}\right)$, where $\boldsymbol{R}_{t}^{1 / 2}$ is the unique positive semi-definite matrix square root [9] of $\boldsymbol{R}_{t}$ and $\boldsymbol{E}=\operatorname{argmin} \operatorname{det}\left(\boldsymbol{E}_{k, l} \boldsymbol{E}_{k, l}^{H}\right)$. For OSTBC, the following property [8] $\left\{\boldsymbol{E}_{k, l}, k \neq l, n\right\}$

holds: $\boldsymbol{E} \boldsymbol{E}^{H}=a d_{\min }^{2} \boldsymbol{I}_{B}$, where $d_{\min }^{2}=\frac{12 \sigma_{x}^{2}}{M^{2}-1}$ for $M$-PAM, where $d_{\min }^{2}=4 \sigma_{x}^{2} \sin ^{2} \frac{\pi}{M}$ for $M$-PSK, and where $d_{\min }^{2}=\frac{6 \sigma_{x}^{2}}{M-1}$ for $M-\mathrm{QAM}$.

An upper bound of the PEP was used as the optimization criterion in [3], however, the criterion that was used was not able to handle singular correlation matrices. In [10], an exact expression was proposed for the SER which could handle singular correlation matrices. Modifying the upper bound expression for the PEP from [3] such that the objective function has a resembling shape to the expressions in [10], we propose to consider the optimal precoder given by the following optimization problem:

\section{Problem 1:}

$$
\begin{aligned}
& \max _{\left\{\boldsymbol{F} \in \mathbb{C}^{M_{t} \times B}\right\}} \operatorname{det}\left(\boldsymbol{I}_{M_{t} M_{r}}+\frac{a d_{\min }^{2}}{4 N_{0}} \boldsymbol{R}^{1 / 2}\left[\left(\boldsymbol{F}^{*} \boldsymbol{F}^{T}\right) \otimes \boldsymbol{I}_{M_{r}}\right] \boldsymbol{R}^{1 / 2}\right) \\
& \text { subject to } a K \sigma_{x}^{2} \operatorname{Tr}\left\{\boldsymbol{F} \boldsymbol{F}^{H}\right\}=P .
\end{aligned}
$$

The objective function in Problem 1 is equivalent to the criterion in [3] when $\boldsymbol{R}$ is non-singular.

\section{B. Properties of the Optimal Precoder}

In this subsection, properties characterizing the optimal precoder in various scenarios are presented. In particular, Theorem 1 allows for a useful simplification of the precoder in the special case of uncorrelated transmitters. Theorem 2 gives the optimal precoder in the special case of Kronecker correlation with uncorrelated transmitters. 
Lemma 1: If $\boldsymbol{F}$ is an optimal solution of Problem 1, then the precoder $\boldsymbol{F} \boldsymbol{U}$, where $\boldsymbol{U} \in \mathbb{C}^{B \times B}$ is unitary, is also optimal.

Proof: Let $\boldsymbol{F}$ be an optimal solution of Problem 1 and $\boldsymbol{U} \in \mathbb{C}^{B \times B}$, be an arbitrary unitary matrix. It is then seen by insertion that the objective function and the power constraint are unaltered by the unitary matrix, hence $\boldsymbol{F} \boldsymbol{U}$ remains optimal in the sense of Problem 1.

Theorem 1: Let $B=M_{t}$. If the transmit antennas are uncorrelated, the autocorrelation matrix is given by (4), then, the optimal $\boldsymbol{F}$ can be chosen diagonal up to a unitary matrix. Without loss of optimality, the precoding matrix can also be chosen real.

Proof: Let the eigenvalue decomposition of $\boldsymbol{R}_{r_{i}}$ be given by $\boldsymbol{R}_{r_{i}}=\boldsymbol{V}_{r_{i}} \boldsymbol{\Lambda}_{r_{i}} \boldsymbol{V}_{r_{i}}^{H}$, where $\boldsymbol{V}_{r_{i}} \in$ $\mathbb{C}^{M_{r} \times M_{r}}$ is unitary and $\boldsymbol{\Lambda}_{r_{i}} \in \mathbb{R}^{M_{r} \times M_{r}}$ is diagonal with non-negative diagonal elements $\lambda_{i_{k}}$. It follows that the eigenvalue decomposition of $\boldsymbol{R}=\boldsymbol{V} \boldsymbol{\Lambda} \boldsymbol{V}^{H}$ is given by the matrices

$$
\boldsymbol{V}=\left[\begin{array}{cccc}
\boldsymbol{V}_{r_{0}} & \mathbf{0}_{M_{r} \times M_{r}} & \cdots & \mathbf{0}_{M_{r} \times M_{r}} \\
\mathbf{0}_{M_{r} \times M_{r}} & \boldsymbol{V}_{r_{1}} & \cdots & \mathbf{0}_{M_{r} \times M_{r}} \\
\vdots & \vdots & \ddots & \vdots \\
\mathbf{0}_{M_{r} \times M_{r}} & \mathbf{0}_{M_{r} \times M_{r}} & \cdots & \boldsymbol{V}_{r_{M_{t}-1}}
\end{array}\right], \boldsymbol{\Lambda}=\left[\begin{array}{cccc}
\boldsymbol{\Lambda}_{r_{0}} & \mathbf{0}_{M_{r} \times M_{r}} & \cdots & \mathbf{0}_{M_{r} \times M_{r}} \\
\mathbf{0}_{M_{r} \times M_{r}} & \boldsymbol{\Lambda}_{r_{1}} & \cdots & \mathbf{0}_{M_{r} \times M_{r}} \\
\vdots & \vdots & \ddots & \vdots \\
& & & \vdots \\
\mathbf{0}_{M_{r} \times M_{r}} & \mathbf{0}_{M_{r} \times M_{r}} & \cdots & \boldsymbol{\Lambda}_{r_{M_{t}-1}}
\end{array}\right] .
$$

The objective function of Problem 1 can now be rewritten as:

$$
\operatorname{det}\left(\boldsymbol{I}_{M_{t} M_{r}}+\frac{a d_{\min }^{2}}{4 N_{0}} \boldsymbol{\Lambda}^{1 / 2} \boldsymbol{V}^{H}\left[\left(\boldsymbol{F}^{*} \boldsymbol{F}^{T}\right) \otimes \boldsymbol{I}_{M_{r}}\right] \boldsymbol{V} \boldsymbol{\Lambda}^{1 / 2}\right) \text {. }
$$

Block element number $(k, l)$ of size $M_{r} \times M_{r}$ of the second addend within the determinant of (7) can be expressed as: $\frac{a d_{\min }^{2}}{4 N_{0}}\left(\boldsymbol{F}^{*} \boldsymbol{F}^{T}\right)_{k, l} \boldsymbol{\Lambda}_{r_{k}}^{1 / 2} \boldsymbol{V}_{r_{k}}^{H} \boldsymbol{V}_{r_{l}} \boldsymbol{\Lambda}_{r_{l}}^{1 / 2}$. Let the second addend within the determinant of (7) be denoted $\boldsymbol{A}$. This matrix is positive semidefinite ${ }^{1}$, and then it follows that $\boldsymbol{I}_{M_{t} M_{r}}+\boldsymbol{A}$ is positive definite. By using Hadamard's inequality [9] on $\operatorname{det}\left(\boldsymbol{I}_{M_{t} M_{r}}+\boldsymbol{A}\right)$, this determinant is maximized when $\boldsymbol{A}$ is diagonal. From the structure of $\boldsymbol{A}$ and due to the fact that the matrices $\boldsymbol{V}_{r_{i}}$ are unitary, it follows that $\boldsymbol{F}^{*} \boldsymbol{F}^{T}$ is diagonal. Hence, $\boldsymbol{F}$ is diagonal up to a unitary matrix, see Lemma 1. Since we can ignore the unitary ambiguity, see Lemma 1, $\boldsymbol{F}$ can be chosen diagonal, real, and non-negative. Thus, it is important to note that optimal precoding in this case 1) is not identity, being instead a function the receive correlation (even though transmitters are uncorrelated!), and 2) boils down to a power allocation strategy over the transmit antennas.

Lemma 2: Let $B=M_{t}$, and let the total correlation matrix be given by (4). Diagonal element number $i$ of the optimal diagonal product $\boldsymbol{F}^{*} \boldsymbol{F}^{T}$ is denoted $f_{i}^{2}$, where $f_{i} \in \mathbb{R}$. Let eigenvalue number $k$

${ }^{1} \boldsymbol{A}$ can be written as $\boldsymbol{A}=\boldsymbol{B} \boldsymbol{B}^{H}$ where $\boldsymbol{B}=\sqrt{\frac{a d_{\min }^{2}}{4 N_{0}}} \boldsymbol{\Lambda}^{1 / 2} \boldsymbol{V}^{H}\left[\boldsymbol{F}^{*} \otimes \boldsymbol{I}_{M_{r}}\right]$, and, therefore, it is positive semidefinite. 
of the correlation matrix $\boldsymbol{R}_{r_{i}}$ be denoted $\lambda_{r_{i_{k}}}$. The optimization problem that must be solved is the following: Find $f_{i}$ such that the following product is maximized:

$$
\prod_{i=0}^{M_{t}-1} \prod_{k=0}^{M_{r}-1}\left(1+\frac{a d_{\min }^{2} f_{i}^{2} \lambda_{r_{i_{k}}}}{4 N_{0}}\right)
$$

subject to

$$
\sum_{i=0}^{M_{t}-1} f_{i}^{2}=\frac{P}{a K \sigma_{x}^{2}}
$$

The proof of this lemma is given in Appendix I.

Interestingly, an analytical precoding method that solves this precoding problem in certain practical situations is presented in a companion publication [10] due to lack of space here.

Theorem 2: Let the correlation model of the channel follow the Kronecker model in (3). If $\boldsymbol{R}_{t}=$ $\boldsymbol{I}_{M_{t}}$, then the optimal precoder is independent of the receiver correlation matrix $\boldsymbol{R}_{r}$ and given by $\boldsymbol{F}=$ $\sqrt{\frac{P}{a K \sigma_{x}^{2} M_{t}}} \boldsymbol{I}_{M_{t}}$.

The proof of this theorem is given in Appendix II.

\section{Iterative Optimization Algorithm}

Unfortunately, in the general case of joint transmit-receive correlation, a closed form solution is difficult to obtain. In this case, a fast-converging iterative algorithm can be proposed in the following theorem.

Theorem 3: Let the matrix $\boldsymbol{K}_{k, l}$ be the commutation ${ }^{2}$ matrix [9] of size $k l \times k l$. The precoder that is optimal for Problem 1 must satisfy

$$
\begin{aligned}
\operatorname{vec}(\boldsymbol{F})= & \mu\left[\boldsymbol{F}^{T} \otimes \boldsymbol{I}_{M_{t}}\right] \boldsymbol{\Pi}\left[\boldsymbol{R}^{1 / 2} \otimes\left(\boldsymbol{R}^{1 / 2}\right)^{T}\right] \\
& \times \operatorname{vec}\left(\left[\boldsymbol{I}_{M_{t} M_{r}}+\frac{a d_{\min }^{2}}{4 N_{0}}\left(\boldsymbol{R}^{1 / 2}\right)^{*}\left[\left(\boldsymbol{F} \boldsymbol{F}^{H}\right) \otimes \boldsymbol{I}_{M_{r}}\right]\left(\boldsymbol{R}^{1 / 2}\right)^{*}\right]^{-1}\right),
\end{aligned}
$$

where $(\cdot)^{*}$ means complex conjugation, $\boldsymbol{\Pi}=\left(\boldsymbol{I}_{M_{t}^{2}} \otimes \operatorname{vec}^{T}\left(\boldsymbol{I}_{M_{r}}\right)\right)\left(\boldsymbol{I}_{M_{t}} \otimes \boldsymbol{K}_{M_{t}, M_{r}} \otimes \boldsymbol{I}_{M_{r}}\right)$, and $\mu$ is a positive scalar chosen such that the power constraint in (5) is satisfied.

The proof of this theorem is given in Appendix III. (10) can now be used in a fixed point iteration for finding the precoder that solves Problem 1.

\footnotetext{
${ }^{2}$ The commutation matrix $\boldsymbol{K}_{k, l}$ is the unique $k l \times k l$ permutation matrix satisfying $\boldsymbol{K}_{k, l} \operatorname{vec}(\boldsymbol{A})=\operatorname{vec}\left(\boldsymbol{A}^{T}\right)$ for all matrices $\boldsymbol{A} \in \mathbb{C}^{k \times l}$.
} 


\section{RESULTS AND COMPARISONS}

In this section, Monte Carlo simulation results are presented for two different non-Kronecker correlation scenarios using 4000000 bits. Since there is no precoder in the literature up to our knowledge capable of handling arbitrary correlation models, comparisons are simply made against a system not employing precoding, i.e., $\boldsymbol{F}=\sqrt{\frac{P}{a K \sigma_{x}^{2} M_{t}}} \boldsymbol{I}_{M_{t}}$. The following parameters are used in the examples: $N=B=M_{t}=2$, $P=1$, and $M_{r} \in\{4,6\}$. The SNR is defined as: $\mathrm{SNR}=10 \log _{10} \frac{P}{N_{0}}$. The signal constellation is Graycoded 4-QAM with $\sigma_{x}^{2}=1 / 2$. The Alamouti code $\boldsymbol{C}(\boldsymbol{x})=\mathcal{G}_{2}^{T}$ is used.

Scenario 1: Let the correlation matrix $\boldsymbol{R}$ be singular and given by (4) with $\boldsymbol{R}_{r_{0}}=\boldsymbol{I}_{M_{r}}$ and $\boldsymbol{R}_{r_{1}}=$ $\mathbf{1}_{M_{r} \times M_{r}}$, where the matrix $\mathbf{1}_{k \times l}$ has size $k \times l$ containing only ones.

Scenario 2: Let the non-Kronecker correlation matrix $\boldsymbol{R}$ be given by $(\boldsymbol{R})_{k, l}=0.99^{|k-l|}$, where the notation $(\cdot)_{k, l}$ picks out element with row number $k$ and column number $l$. Although this is not necessarily a practical case, this serves as a test-case for the proposed algorithm.

Results: Figures 2 and 3 show the BER versus SNR performance for the non-precoded reference system and the proposed precoded system when the trivial identity-based precoder was used as the initial value in the fixed-point iteration. From the figures, it is seen that proposed precoder outperforms the reference system. For Scenario 1, the performance gain is bigger for $M_{r}=6$ than for $M_{r}=4$ and for Scenario 2, it is bigger for low values of SNR. It is observed from the results that the gain of precoding is lost when SNR approaches infinity. This can also be observed from the objective function since when $N_{0} \rightarrow 0^{+}$, the term inside the determinant containing $N_{0}$ will be dominating, and by utilizing rules for the determinant of a matrix- and Kronecker- product and the Hadamard inequality, it is seen that as $N_{0} \rightarrow 0^{+}$, the optimal precoder will be proportional to the identity matrix. In all cases, the gain due to precoding (about two $\mathrm{dB})$ in the presence of receive correlation appears clearly.

Figure 4 shows the empirical convergence rate for $\mathrm{SNR}=8 \mathrm{~dB}$ for Scenario 2 with $M_{r}=4$. It is seen from the plot that the algorithm converges very fast. Typically, within one to two iterations the algorithm has converged to a satisfactory performing precoder. In Figure 4, the algorithm converged after one iteration.

\section{CONCLUSIONS}

A precoder is proposed that minimizes a certain PEP based metric for transmission of OSTBC over flat block-fading correlated Rayleigh MIMO channels. Several features of the optimal solution were derived for 
special cases. For the case where the autocorrelation matrix is given, a fast converging iterative numerical optimization technique was proposed.

\section{APPENDIX I}

\section{PROOF OF LEMMA 2}

Proof: Under the assumptions in the lemma, the determinant on (7) can be written as

$$
\operatorname{det}\left(\boldsymbol{I}_{M_{t} M_{r}}+\frac{a d_{\min }^{2}}{4 N_{0}} \boldsymbol{\Lambda}^{1 / 2} \boldsymbol{V}^{H}\left[\left(\boldsymbol{F}^{*} \boldsymbol{F}^{T}\right) \otimes \boldsymbol{I}_{M_{r}}\right] \boldsymbol{V} \boldsymbol{\Lambda}^{1 / 2}\right)=\prod_{i=0}^{M_{t}-1} \operatorname{det}\left(\boldsymbol{I}_{M_{r}}+\frac{a d_{\min }^{2} f_{i}^{2}}{4 N_{0}} \boldsymbol{\Lambda}_{r_{i}}\right) \text {. }
$$

The right hand side of (11) can be rewritten as the objective function shown in (8). The power constraint reformulation follows directly by inserting the diagonal matrix product $\boldsymbol{F} \boldsymbol{F}^{H}$ into (5).

\section{APPENDIX II}

\section{ProOF OF THEOREM 2}

Proof: If the Kronecker model holds, then $\boldsymbol{R}_{r_{i}}=\boldsymbol{R}_{r}$. From Lemma 2, it follows that the unconstrained Lagrange function for the problem that should be solved can be written as

$$
\sum_{i=0}^{M_{t}-1}\left(\ln \operatorname{det}\left(\boldsymbol{I}_{M_{r}}+\frac{a d_{\min }^{2} f_{i}^{2}}{4 N_{0}} \boldsymbol{\Lambda}_{r}\right)+\mu f_{i}^{2}\right)
$$

where $\mu$ is a Lagrange multiplier for the power constraint. It is seen that the unconstrained objective function in (12) is symmetric in the variables $f_{i}$. Therefore, in the optimum solution of the maximization problem, all the independent variables $f_{i}$ must be equal. This leads to the following optimal precoder $\boldsymbol{F}=$ $\sqrt{\frac{P}{a K \sigma_{x}^{2} M_{t}}} \boldsymbol{I}_{M_{t}}$.

\section{APPENDIX III}

\section{Proof of TheOREM 3}

Proof: The constrained maximization Problem 1 can be converted into an unconstrained optimization problem by introducing a Lagrange multiplier $\mu$. This is done by defining the following Lagrange function:

$$
\mathcal{L}(\boldsymbol{F})=\operatorname{det}\left(\boldsymbol{I}_{M_{t} M_{r}}+\frac{a d_{\min ^{2}}}{4 N_{0}} \boldsymbol{R}^{1 / 2}\left[\left(\boldsymbol{F}^{*} \boldsymbol{F}^{T}\right) \otimes \boldsymbol{I}_{M_{r}}\right] \boldsymbol{R}^{1 / 2}\right)-\mu^{\prime} \operatorname{Tr}\left\{\boldsymbol{F} \boldsymbol{F}^{H}\right\}
$$

Since the objective function should be maximized, $\mu^{\prime}$ should be positive.

The necessary condition for the optimality of Problem 1 is found by setting the derivative of the Lagrangian in (13) with respect to vec $\left(\boldsymbol{F}^{*}\right)$ equal to the zero vector of the same size. Finding the derivative with respect to the complex valued vector $\operatorname{vec}\left(\boldsymbol{F}^{*}\right)$ can be done by generalizing the works in [9], [11] 
when the differentials of $\boldsymbol{F}$ and $\boldsymbol{F}^{*}$ are treated as independent. The following two expressions, which are found after several matrix manipulations, are useful for finding the necessary conditions:

$$
\frac{\partial}{\partial \operatorname{vec}\left(\boldsymbol{F}^{*}\right)} \operatorname{Tr}\left\{\boldsymbol{F} \boldsymbol{F}^{H}\right\}=\operatorname{vec}(\boldsymbol{F}) \text {, }
$$

and

$$
\begin{aligned}
& \frac{\partial}{\partial \operatorname{vec}\left(\boldsymbol{F}^{*}\right)} \operatorname{det}\left(\boldsymbol{I}_{M_{t} M_{r}}+\frac{a d_{\min }^{2}}{4 N_{0}} \boldsymbol{R}^{1 / 2}\left[\left(\boldsymbol{F}^{*} \boldsymbol{F}^{T}\right) \otimes \boldsymbol{I}_{M_{r}}\right] \boldsymbol{R}^{1 / 2}\right)= \\
& \operatorname{det}\left(\boldsymbol{I}_{M_{t} M_{r}}+\frac{a d_{\min }^{2}}{4 N_{0}} \boldsymbol{R}^{1 / 2}\left[\left(\boldsymbol{F}^{*} \boldsymbol{F}^{T}\right) \otimes \boldsymbol{I}_{M_{r}}\right] \boldsymbol{R}^{1 / 2}\right) \frac{a d_{\min }^{2}}{4 N_{0}}\left[\boldsymbol{F}^{T} \otimes \boldsymbol{I}_{M_{t}}\right] \boldsymbol{\Pi} \\
& \times\left[\left(\boldsymbol{R}^{1 / 2}\right)^{*} \otimes \boldsymbol{R}^{1 / 2}\right] \operatorname{vec}\left(\left(\boldsymbol{I}_{M_{t} M_{r}}+\frac{a d_{\min }^{2}}{4 N_{0}}\left(\boldsymbol{R}^{1 / 2}\right)^{*}\left[\left(\boldsymbol{F}^{*} \boldsymbol{F}^{T}\right) \otimes \boldsymbol{I}_{M_{r}}\right]\left(\boldsymbol{R}^{1 / 2}\right)^{*}\right)^{-1}\right),
\end{aligned}
$$

where the $M_{t}^{2} \times M_{t}^{2} M_{r}^{2}$ matrix $\boldsymbol{\Pi}$ is defined in the theorem statement. The necessary conditions for optimality can be found by utilizing the results from (14) and (15) and setting the derivative of the Lagrangian multiplier in (13) equal to zero. If this is done, and scalar factors are collected into the scalar named $\mu$, the result in (10) is found. Since the precoder matrix $\boldsymbol{F}$ should be scaled according to (5), it is not necessary to decide the exact value of the scalar $\mu$. This scalar can be found by adjusting the norm of the precoder according to (5) after each time the fixed point iteration is used.

\section{REFERENCES}

[1] A. Hjørungnes, J. Akhtar, and D. Gesbert, "Precoding for space-time block codes in (non-)Kronecker correlated MIMO channels," in Proc. of Eur. Signal Proc. Conf., Vienna, Austria, Sept. 2004, pp. 515-518, invited paper to special session on 'Performance limits and signal design for MIMO wireless'.

[2] A. Paulraj, R. Nabar, and D. Gore, Introduction to Space-Time Wireless Communications. Cambridge, United Kingdom: Cambridge University Press, May 2003.

[3] G. Jöngren, M. Skoglund, and B. Ottersten, "Combining beamforming and orthogonal space-time block coding," IEEE Trans. Inform. Theory, vol. 48, no. 3, pp. 611-627, Mar. 2002.

[4] H. Sampath and A. Paulraj, "Linear precoding for space-time coded systems with known fading correlations," IEEE Communications Letters, vol. 6, no. 6, pp. 239-241, June 2002.

[5] D. Gesbert, M. Shafi, D. Shiu, P. J. Smith, and A. Naguib, "From theory to practice: An overview of MIMO space-time coded wireless systems,” IEEE J. Select. Areas Commun., vol. 21, no. 3, pp. 281-302, Apr. 2003.

[6] L. Liu and H. Jafarkhani, "Combining beamforming and quasi-orthogonal space-time block coding using channel mean feedback," in Proc. IEEE GLOBECOM, vol. 4, San Fransico, USA, Dec. 2003, pp. 1925-1930.

[7] E. Bonek, H. Özcelik, M. Herdin, W. Weichselberger, and J. Wallace, "Deficiencies of a popular stochastic MIMO radio channel model," in Proc. Int. Symp. on Wireless Personal Multimedia Communications, Yokosuka, Japan, Oct. 2003.

[8] V. Tarokh, H. Jafarkhani, and A. R. Calderbank, "Space-time block coding for wireless communications: Performance results," IEEE J. Select. Areas Commun., vol. 17, no. 3, pp. 451-460, Mar. 1999.

[9] J. R. Magnus and H. Neudecker, Matrix Differential Calculus with Application in Statistics and Econometrics. Essex, England: John Wiley \& Sons, Inc., 1988.

[10] A. Hjørungnes and D. Gesbert, "Minimum exact SER precoder for orthogonal space-time block codes over correlated Rayleigh MIMO channels," IEEE Trans. Wireless Communications, to be submitted.

[11] D. H. Brandwood, "A complex gradient operator and its application in adaptive array theory," IEE Proc., Parts F and H, vol. 130, no. 1, pp. 11-16, Feb. 1983. 


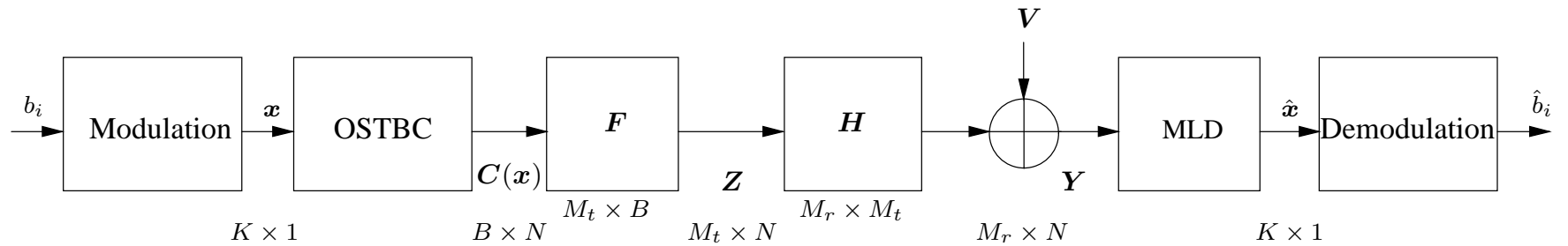

Fig. 1. Block model of the linear precoded OSTBC MIMO system.
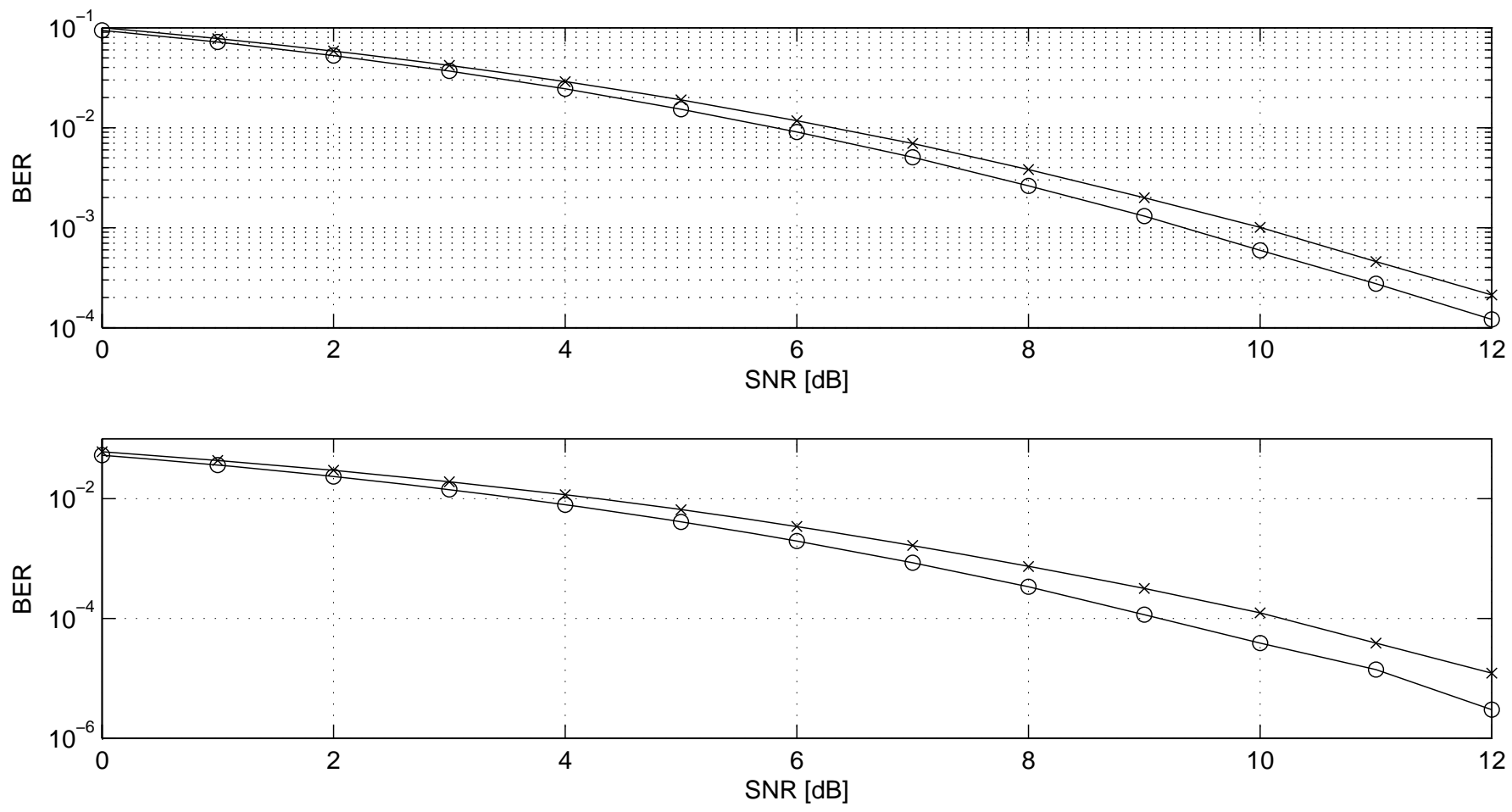

Fig. 2. Scenario 1: BER versus SNR performance of the proposed system - o - using the numerical optimization method in Theorem 3 and a system not employing a precoder $-\times-$. In the upper plot, $M_{r}=4$ and in the lower plot $M_{r}=6$. 

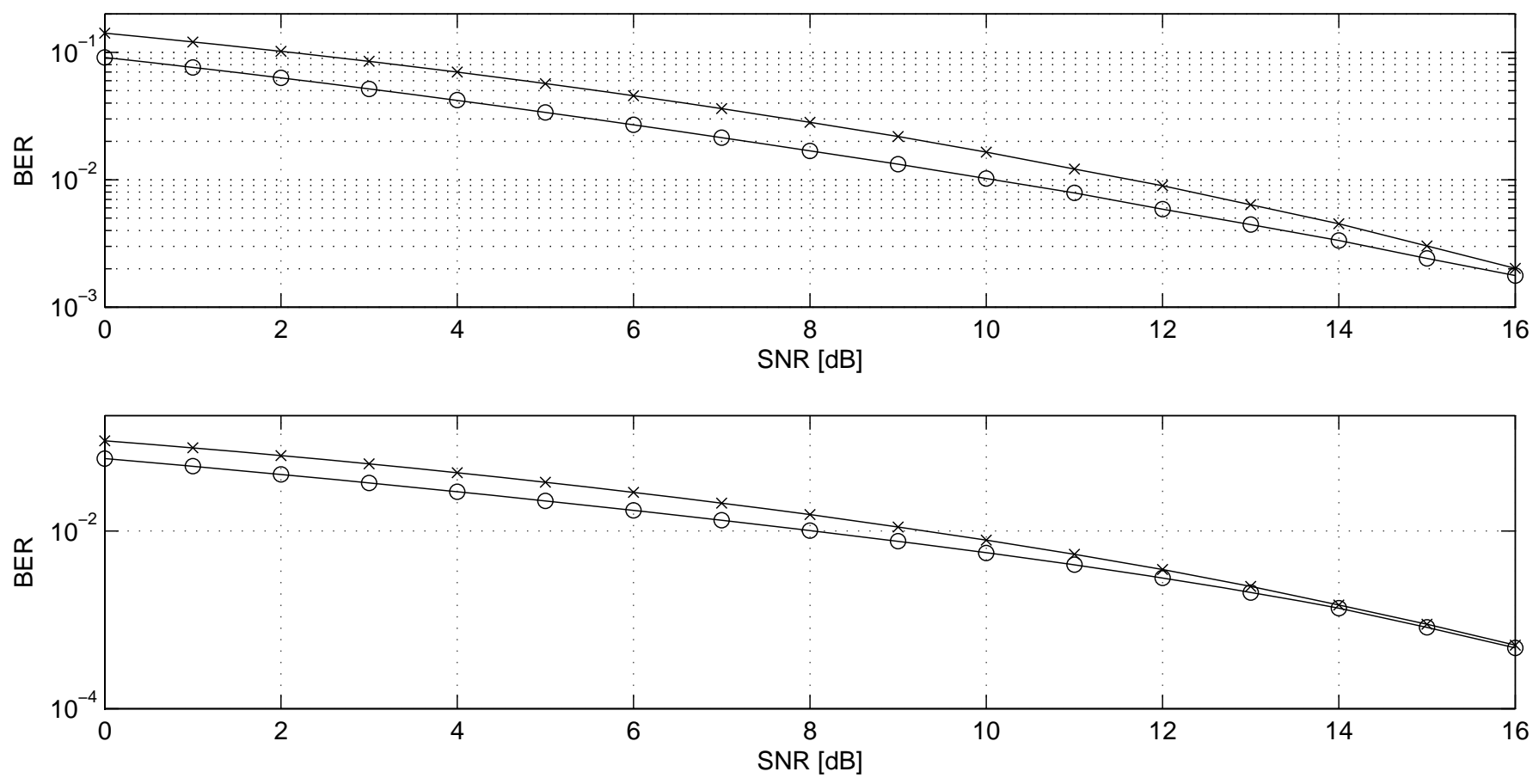

Fig. 3. Scenario 2: BER versus SNR performance of the proposed system - o - using the numerical optimization method in Theorem 3 and a system not employing a precoder $-\times-$. In the upper plot, $M_{r}=4$ and in the lower plot $M_{r}=6$.

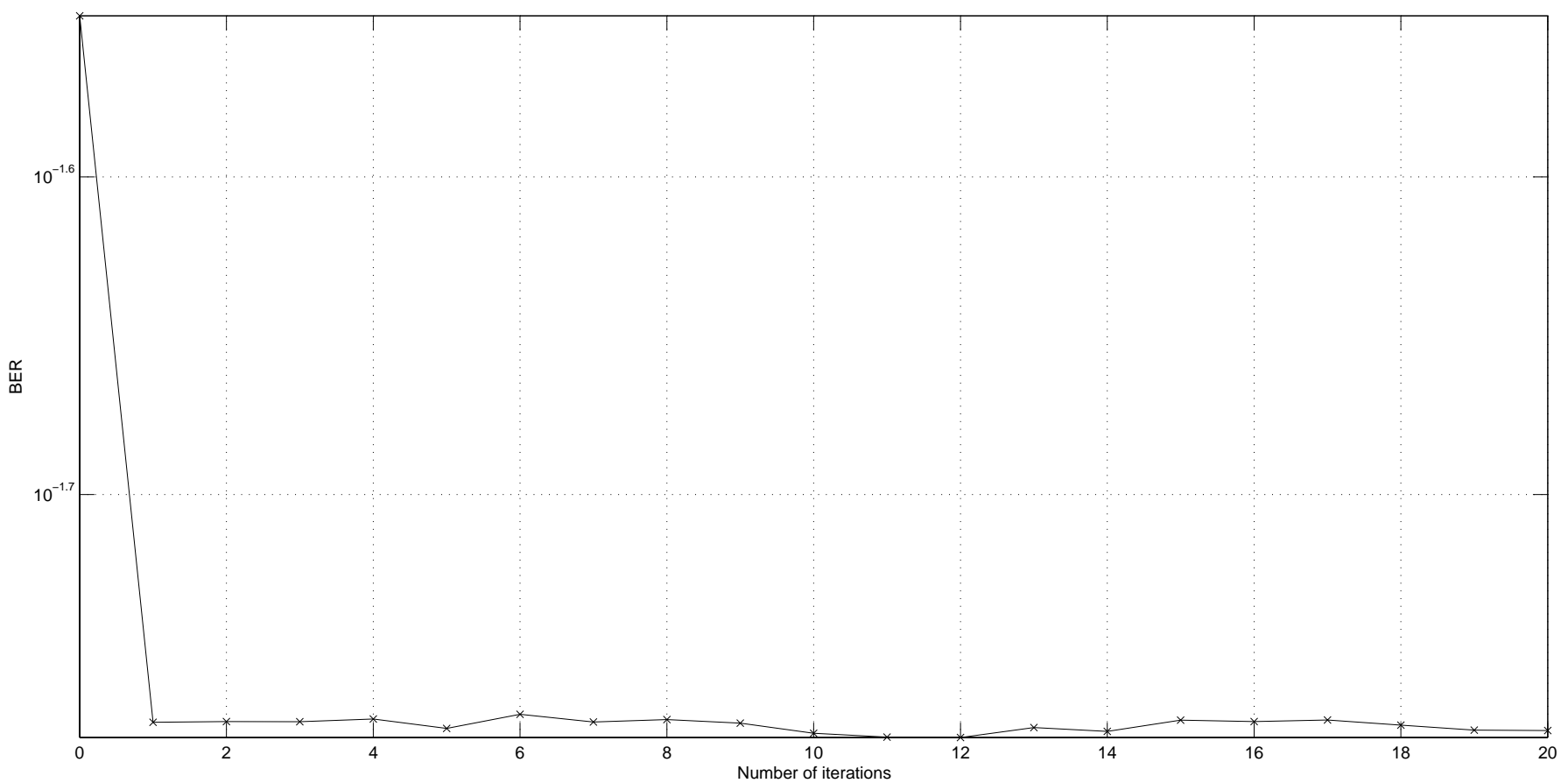

Fig. 4. Empirical convergence for Scenario 2, $\mathrm{SNR}=8 \mathrm{~dB}, M_{r}=4$. Zero iteration correspond to the system not employing a precoder. 